\title{
Research on Soft Fault Diagnosis of Wavelet Neural Network Based on UKF Algorithm for Analog Circuit
}

\author{
Yan-ming Wei ${ }^{1, \mathrm{a}}$,Bao-Hua Zhang ${ }^{2, \mathrm{~b}}$,Wen-wen You $^{2, \mathrm{c}}$ \\ ${ }^{1}$ XiJing College, Xi’an, Shaanxi, 710123, China \\ ${ }^{2}$ Air Traffic Control and Navigation College, Air Force Engineering \\ University, Xi'an, Shaanxi, 710051, China \\ a ganxusheng123@163.com, ${ }^{b}$ zhangbh6768@163.com, cyww123@163.com
}

Keywords: Wavelet; Neural network; Unscented Kalman filter; Fault diagnosis; Analog circuit

\begin{abstract}
In order to improve the capacity of soft fault diagnosis for analog circuit, a Wavelet Neural Network (WNN) diagnosis method based on Unscented Kalman Filter (UKF) algorithm is proposed. To overcome the shortcoming of the traditional BP algorithm of WNN, UKF algorithm is introduced to optimize the parameters of WNN, completing the training of WNN. The simulation shows that the proposed modeling algorithm has the better diagnosis capacity. This also verifies the feasible and effectiveness of proposed method.
\end{abstract}

\section{Introduction}

Currently, a focus for concern is the issues about soft fault diagnosis of analog circuit caused by the tolerance etc. that easily deteriorates the performance of circuit and system. To ensure the reliability and integrity of electronic equipment, the soft fault in analog circuit need to be diagnosed and located accurately for the prevention and recovery.

Research has shown that, when a soft fault occurs in analog circuit, the deviation of parameter values can have a different effect on the circuit and can bring large numbers of circuit states, which cause severe calculation hardship for the traditional methods [1][2]. However, the occurrence of Neural Network (NN) completely converts the original modes of fault diagnosis for analog circuit [3][4]. In the past decades, NN has been a hot topic in fault diagnosis research for analog circuit, and can be competent for the fault diagnosis task well even for the nonlinear analog circuit without the explicit model. The common ground of these studies is that, for the difficulties to model the complex relationship between input and output of analog circuit, using fault features extracted by wavelet transform, the advantages of $\mathrm{NN}$, such as simplicity and adaptive learning without requiring to determine the intrinsic relationship between input and output, are applied to build the diagnosis model, overcoming the drawbacks of traditional diagnosis method. However, due to its own reason of optimization algorithm of $\mathrm{NN}$ and difficulty brought by component tolerance in analog circuit, the diagnosis accuracy and efficiency gotten by these NNs cannot still satisfy the practical applications. In spite of many improvements for it, the problem cannot be solved fundamentally. Wavelet Neural Network (WNN) is also an ideal substitute for traditional NN [5][6]. It can overcome many disadvantages of conventional NN, and, which provides the necessary condition for fault diagnosis in analog circuit.

According to above analysis, a WNN based on UKF algorithm is proposed to improve the soft fault diagnosis ability of WNN model in analog circuit. The simulation results verify its validity and feasibility. 


\section{Wavelet neural network}

WNN is a novel neural network derived from wavelet transform. It integrates the time-frequency localization of wavelet transform with self-learning function of neural network [5][7]. Compared with conventional neural network, WNN has a good improvement in accuracy, convergence and fault tolerance in modeling the nonlinear system.

Considering the discrete wavelet transform, through the wavelet function

$$
\psi_{m, n}(t)=2^{-\frac{m}{2}} \psi\left(2^{-m} t-n\right), \quad m, n \in Z
$$

a series of orthogonal basis in the space $L^{2}(R)=\underset{m}{\oplus} W_{m}$, can be obtained. Through the multi-resolution analysis in $L^{2}(R)$, a series of closed subspaces $\cdots \subset V_{-2} \subset V_{-1} \subset V_{0} \subset V_{1} \subset V_{2} \cdots$ can also be obtained where $V_{m}=\operatorname{span}\left\{2^{-m / 2} \varphi\left(2^{-m} t-k\right)\right\}, V_{m+1}=V_{m} \oplus W_{m}$, and $\varphi(t)$ is the scale function of corresponding wavelet. $f(t)$ in $L^{2}(R)$ can be decomposed into

$$
f(t)=\sum_{m, n}\left\langle f, \psi_{m, n}\right\rangle \psi_{m, n}(t)
$$

In depth, this formula can also be described as

$$
\hat{f}=\sum_{m, n}^{N} w_{m, n} \psi_{m, n}(t)=\sum_{m, n}^{N} w_{m, n} 2^{-\frac{m}{2}} \psi\left(2^{-m} t-n\right)
$$

where $\psi_{m, n}(t)$ is the orthogonal wavelet function, and used as the activation function in hidden node. $t_{j}=\sum_{i=1}^{I} w_{j i} x_{i}$. In addition, $w_{j i}$ is the connection weights from input layer to hidden layer, $w_{k j}$ is the connection weights from the hidden layer to the output layer. The formula (3) can be described by structure of WNN as shown in Fig. 1.

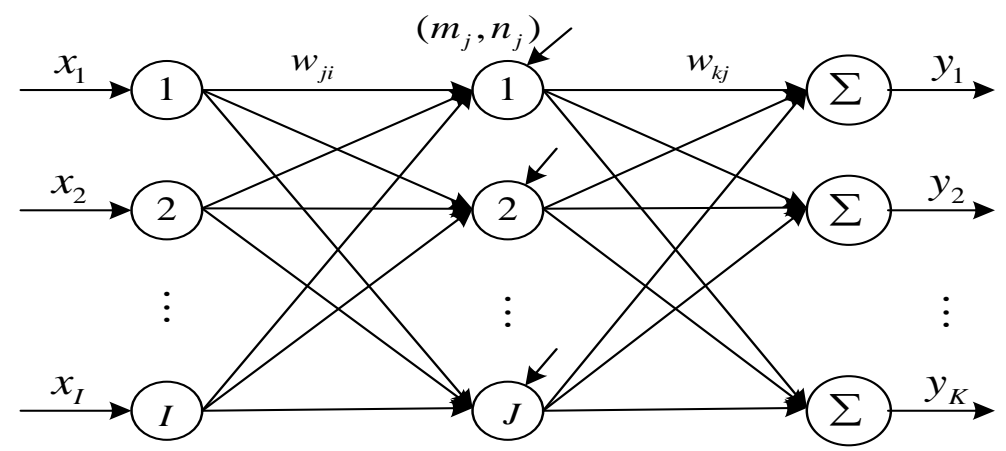

Fig. 1 Structure of WNN

In fact, the training process of WNN mainly refers to the optimization process of parameter vector $\theta_{k}\left(w_{j i}, w_{k j}, m_{j}, n_{j}\right)$ of WNN. Generally, by using the different optimization algorithm for the parameters, the different training algorithm of WNN can be obtained. In state space model of WNN, the parameters vector $\theta_{k}$ can be considered as the state variables, the output of network can be considered as the observation variables. Then the state space model of WNN can be expressed as

$$
\begin{aligned}
& \theta_{k}=\theta_{k-1}+\eta_{k} \\
& y_{k}=h\left(\theta_{k}, u_{k}\right)+\mu_{k}
\end{aligned}
$$

where $\eta_{k}$ is the process noise that is the white Gaussian noise with mean 0 and variance $Q_{k}$. $y_{k}$ denotes the output of network. $h\left(\theta_{k}, u_{k}\right)$ is the nonlinear function parameterized. $u_{k}$ denotes the input of network. $\mu_{k}$ is the measure noise that is the white Gaussian noise with mean 0 and variance $R_{k}$. 


\section{UKF algorithm}

UKF algorithm proposed by Julier is a Kalman filter algorithm based on approximate distribution. UT (Unscented Transformation) is the core of the algorithm. It uses the deterministic sampling strategy to approximate the nonlinear distribution and can estimate the posteriori mean and covariance of the nonlinear system with higher accuracy [8].

Suppose that the state equation and the observation equation are, respectively

$$
\begin{aligned}
& x_{k+1}=f\left(x_{k}\right)+\omega_{k} \\
& y_{k}=g\left(x_{k}, u_{k}\right)+v_{k}
\end{aligned}
$$

where $\omega_{k}$ is the process noise with the covariance matrix $Q_{k} \cdot v_{k}$ is the measure noise with the covariance matrix $R_{k} \cdot \omega_{k}$ and $v_{k}$ are the white Gaussian noise with mean 0 , and $\omega_{k}$ is irrelevant to $v_{k}$.

The realization steps of UKF algorithm are as follows.

1. Initialization

$$
\begin{gathered}
\hat{x}_{0}=E\left[x_{0}\right] \\
P_{0}=E\left[\left(x_{0}-\hat{x}_{0}\right)\left(x_{0}-\hat{x}_{0}\right)^{\mathrm{T}}\right]
\end{gathered}
$$

2. Sigma point sampling and weight determination

$$
\begin{aligned}
& \chi_{i, k \mid k}=\left[\begin{array}{lll}
\hat{x}_{k} & \hat{x}_{k}+\left(\sqrt{(n+\lambda) P_{k}}\right)_{i} & \hat{x}_{k}-\left(\sqrt{(n+\lambda) P_{k}}\right)_{i}
\end{array}\right] \\
& \left\{\begin{array}{l}
W^{m}=\lambda /(n+\lambda) \\
W_{0}^{c}=\lambda /(n+\lambda)+\left(1-\eta^{2}+\beta\right) \\
W_{i}^{m}=W_{i}^{c}=0.5 /(n+\lambda)
\end{array}\right.
\end{aligned}
$$

where $\chi_{i, k \mid k}$ denote the Sigma point. $\hat{x}_{k}$ is the posteriori estimate of state vector at time $k$. $i=1, \cdots, 2 n+1$, and $2 n+1$ is the number of samples for Sigma point, $n$ is the dimension of random variable. $W_{i}^{m}$ and $W_{i}^{c}$ are the weights of mean and variance of Sigma point, respectively, the scale parameter $\lambda=\alpha^{2}(n+\tau)-n$. $\alpha$ is used to control the distance from Sigma point to the center point and $10^{-4} \leq \alpha \leq 1$. The other scale parameter $\tau=3-n$. $\beta$ is used to incorporate pre-test information for random variables, for Gaussian distributions, $\beta=2$ is optimal.

3. Time update

$$
\begin{gathered}
\chi_{i, k+1 \mid k}=f\left(\chi_{i, k \mid k}\right) \\
\hat{x}_{k}^{-}=\sum_{i=0}^{2 n} W_{i}^{m} \chi_{i, k+1 \mid k} \\
P_{k}^{-}=\sum_{i=0}^{2 n} W_{i}^{c}\left(\chi_{i, k+1 \mid k}-\hat{x}_{k}^{-}\right)\left(\chi_{i, k+1 \mid k}-\hat{x}_{k}^{-}\right)^{\mathrm{T}}+Q_{k+1}
\end{gathered}
$$

where, $\hat{x}_{k}^{-}$and $P_{k}^{-}$are the prediction mean and variance of the state variables, respectively.

4. Sampling point of prediction measurement 。

$$
\begin{aligned}
& y_{i, k+1 \mid k}=g\left(\chi_{i, k+1 \mid k}, u_{k}\right) \\
& \hat{y}_{k}^{-}=\sum_{i=0}^{2 n} W_{i}^{m} y_{i, k+1 \mid k}
\end{aligned}
$$

5. Measure update

$$
\begin{aligned}
P_{y y} & =\sum_{i=0}^{2 n} W_{i}^{c}\left(y_{i, k+1 \mid k}-\hat{y}_{k}^{-}\right)\left(y_{i, k+1 \mid k}-\hat{y}_{k}^{-}\right)^{\mathrm{T}}+R_{k+1} \\
P_{x y} & =\sum_{i=0}^{2 n} W_{i}^{c}\left(\chi_{i, k+1 \mid k}-\hat{x}_{k}^{-}\right)\left(y_{i, k+1 \mid k}-\hat{y}_{k}^{-}\right)^{\mathrm{T}}
\end{aligned}
$$


where $y_{i, k+1 \mid k}$ is the estimated value of the observation vector. $P_{y y}$ is the covariance matrix of the observation vector. $P_{x y}$ is the covariance matrix of the state vector and the observation vector.

6. State update

$$
\begin{gathered}
K_{k+1}=P_{x y} \cdot P_{y y}^{-1} \\
\hat{x}_{k+1}=\hat{x}_{k}^{-}+K_{k+1}\left(y_{k+1}-\hat{y}_{k}^{-}\right) \\
P_{k+1}=P_{k}^{-}-K_{k+1} P_{y y} K_{k+1}^{\mathrm{T}}
\end{gathered}
$$

where $K_{k+1}$ is Kalman gain matrix.

\section{Simulation example}

Sallen-Key bandpass filter is selected as the research subject of fault diagnosis for analog circuit. The nominal value of each component in the circuit is shown in Fig. 2. The normal tolerance range of resistance is set as $5 \%$. The normal tolerance range of capacitance is set as $10 \%$. The output "Out” is only test node. The paper only studies the single soft fault of resistance and capacitance without.

ORCAD is used to simulate the circuit. Through AC small-signal sensitivity analysis, the sensitivity of the influence of each component on output waveform is compared by way of summary. It can be known that the change of $R_{2}, R_{3}, C_{1}, C_{2}$ has the greatest impact on the output response waveform of the circuit. Therefore, it can determine that the fault set contains 8 fault modes, namely $\mathrm{R}_{2^{-}}, \mathrm{R}_{2}+, \mathrm{R}_{3^{-}}, \mathrm{R}_{3}+, \mathrm{C}_{1^{-}}, \mathrm{C}_{1}+, \mathrm{C}_{2^{-}}, \mathrm{C}_{2}+$, where the symbol '+' and '-' represents too large soft fault and too small soft fault respectively. All nominal value of deviation error are set as $\pm 50 \%$. In this way 8 fault modes together with normal mode add up to 9 modes. In addition, "0-l" method can be used to obtain the expected output vector of 8 output nodes in UKF-WNN, as shown in Table 1.

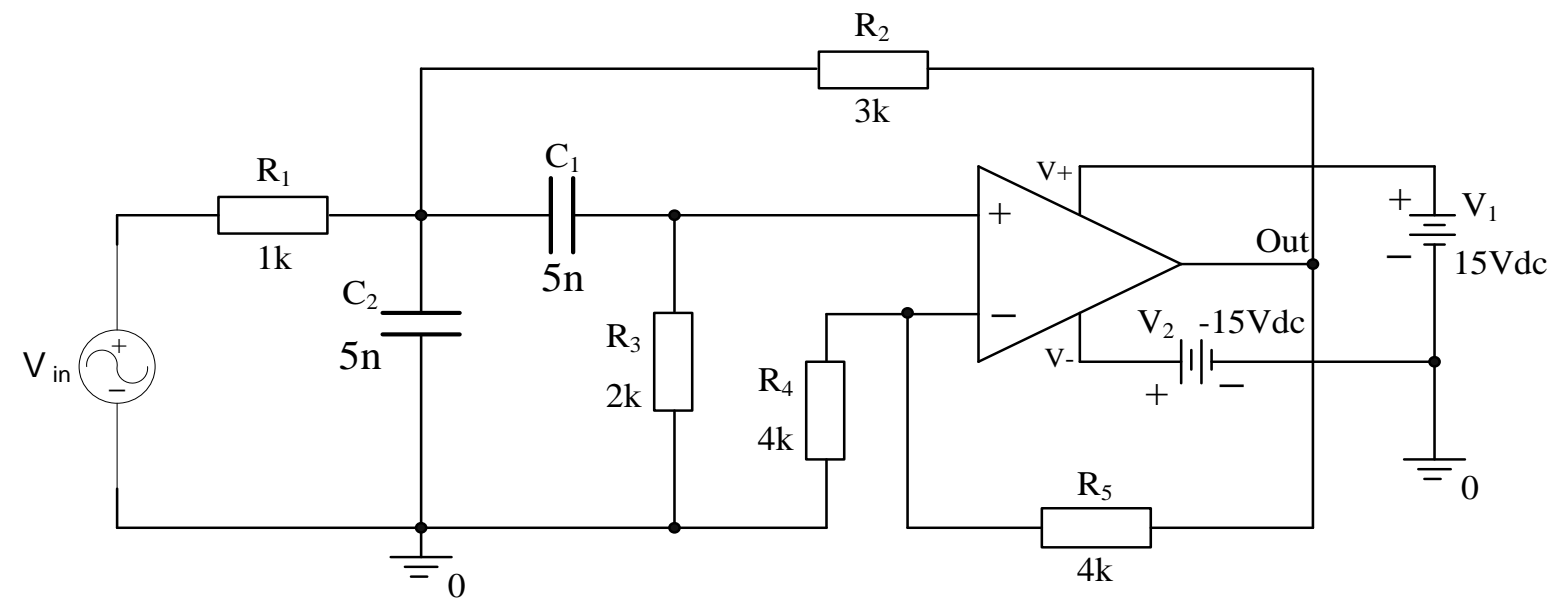

Fig. 2 Sallen-Key bandpass filter

To verify the proposed method, the diagnosis results of UKF-WNN needs to be compared with that of BP-NN and BP-WNN in fault diagnosis test for the selected circuit. The parameters $w_{j i}, w_{k j}, m_{j}, n_{j}$ can initially be taken as the random number in $[-1,1]$. The number of hidden nodes of WNN can be determined as 20 by empirical formula $J \geq \sqrt{I+K}+d$ ( $d$ is a integer in $[1,10]$ ). The wavelet basis function can be selected as Morlet wavelet function $\psi(t)=\cos (1.75 t) e^{-t^{2} / 2}$. Maximum number of training can be set as 500. In the experiment, if the absolute value of difference between expected output and actual output of an output node in WNN is greater than the determining value 0.3 , then it can be considered that the diagnosis result is incorrect. For UKF algorithm, the value of covariance matrix $R_{k}$ of measure noise has little effect on the result, the diagonal components of $R_{k}$ can be taken as 0.0001 . the diagonal components of $Q_{k}$ can be taken as 0.1 .

The modeling and diagnosis result of BP-NN, BP-WNN, and UKF-WNN is compared in Table 1. The correct diagnosis results of 2 built models for each mode are shown in Table 2, where $\mathrm{C}$ 
represents the number of correct diagnosis, $\mathrm{R}$ represents the correct diagnosis rate. The training convergence curves of UKF-WNN modeling is given in Fig. 3, where MSE denotes the root mean square error of th training.

It can be seen from the experiment results that, for soft fault diagnosis for analog circuits, compared with BP-NN and BP-WNN, UKF-WNN have a more obvious diagnostic advantage. In 2 WNNs, UKF-WNN is better than BP-WNN in Training Misclassification Number (TrMN), Test Misclassification Number (TeMN) and Convergence Times (CT). This shows that UKF algorithm have authentically the superiority over traditional BP algorithm in WNN modeling. This is very important for the wider application of UKF-WNN in soft fault diagnosis for analog circuit.

Table 1 Comparison of modeling capability of 3 algorithms

\begin{tabular}{ccccc}
\hline $\begin{array}{c}\text { Hidden nodes } \\
\text { number }\end{array}$ & $\begin{array}{c}\text { Diagnosis } \\
\text { algorithm }\end{array}$ & TrMN & TeMN & CT \\
\hline 10 & BP-NN & 37 & 52 & 500 \\
& BP-WNN & 21 & 28 & 478 \\
& UKF-WNN & 15 & 19 & 212 \\
\hline 15 & BP-NN & 35 & 42 & 500 \\
& BP-WNN & 22 & 25 & 334 \\
& UKF-WNN & 10 & 16 & 152 \\
\hline & BP-NN & 45 & 64 & 500 \\
& BP-WNN & 25 & 31 & 428 \\
\hline
\end{tabular}

Table 2 Comparison of correct diagnosis result of 2 models

\begin{tabular}{|c|c|c|c|c|c|c|c|c|c|c|c|}
\hline Fault mode & & $\mathrm{R}_{2^{-}}$ & $\mathrm{R}_{2}+$ & $\mathrm{R}_{3^{-}}$ & $\mathrm{R}_{3}+$ & $\mathrm{C}_{1^{-}}$ & $\mathrm{C}_{1}+$ & $\mathrm{C}_{2^{-}}$ & $\mathrm{C}_{2}+$ & Normal & Total \\
\hline Sample number & & 32 & 30 & 28 & 27 & 29 & 29 & 32 & 32 & 31 & 270 \\
\hline \multirow{2}{*}{ BP-WNN } & $\mathrm{C}$ & 27 & 24 & 23 & 23 & 25 & 24 & 27 & 29 & 27 & 229 \\
\hline & $\mathrm{R}$ & $84.4 \%$ & $80.0 \%$ & $82.1 \%$ & $85.2 \%$ & $86.2 \%$ & $82.8 \%$ & $84.4 \%$ & $90.6 \%$ & $87.1 \%$ & $84.8 \%$ \\
\hline \multirow{2}{*}{ UKF-WNN } & $\mathrm{C}$ & 29 & 26 & 25 & 24 & 26 & 25 & 28 & 30 & 29 & 242 \\
\hline & $\mathrm{R}$ & $90.6 \%$ & $86.7 \%$ & $89.3 \%$ & $88.9 \%$ & $89.7 \%$ & $86.2 \%$ & $87.5 \%$ & $93.8 \%$ & $93.6 \%$ & $89.6 \%$ \\
\hline
\end{tabular}




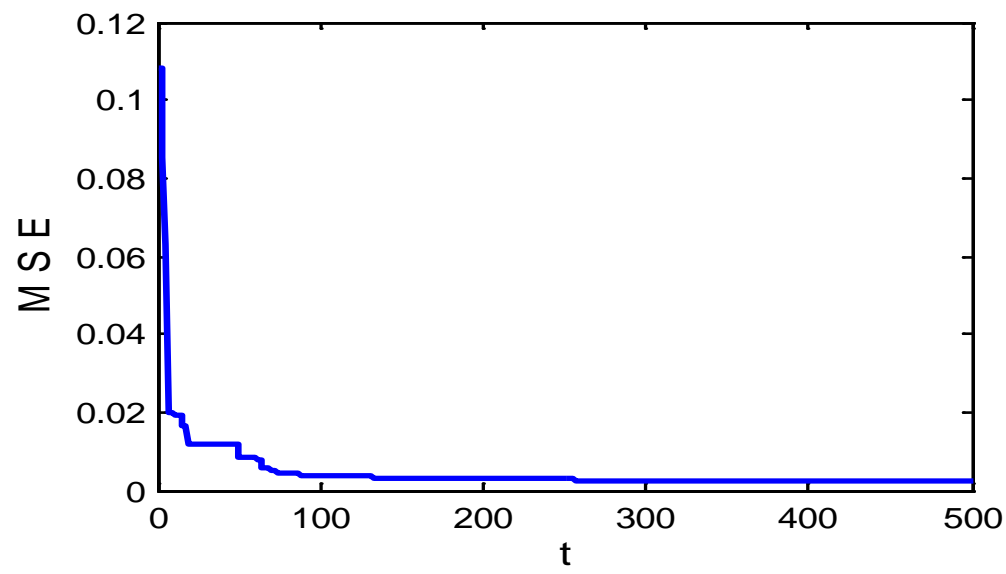

Fig.3 Convergence curve of UKF-WNN modeling

\section{Conclusions}

A WNN soft fault diagnosis method based on UKF algorithm is proposed for analog circuits. In this diagnosis method, UKF algorithm is used to optimizationally estimate the parameters of WNN diagnosis model. The simulation results on Sallen-Key bandpass filter shows that, the proposed diagnosis method has fast convergence rate and high diagnosis accuracy, greatly improving the diagnosis performance of soft fault, which provides a new approach for so fault diagnosis for analog circuit.

\section{References}

[1] J. Huang, Y. G. He. The state-of-the-art of fault diagnosis of analog circuits and prospect. Microelectronics, 34(1), (2004), 21-25.

[2] T. Xie, Y. He. Fault diagnosis of analog circuit based on high-order cumulants and information fusion. Journal of Electronic Testing, 30(5), (2014), 505-514.

[3] Tan Y H, He Y G. Wavelet method for fault diagnosis of analogue circuits. Transactions of China Electro-technical Society, 20(8), (2005), 89-93.

[4] J. J. Zhou, H. H. Cheng, M. An, et a1. Research on analog circuit fault diagnosis based on neural network method. Modern Electronics Technique, 23, (2015), 47-50.

[5] Q. H. Zhang, Benveniste A. Wavelet network. IEEE Transactions on Neural Networks, 3(6), (1992), 889-898.

[6] Preseren P P, Stopar B. Wavelet neural network employment for continuous GNSS orbit function construction: application for the assisted-GNSS principle. Applied Soft Computing, 13(5), (2013), 2526-2536.

[7] Q. H. Zhang. Using wavelet network in nonparametric estimation. IEEE Transactions on Neural Networks, 8(2), (1997), 227-236.

[8] S. J. Julier, J. K. Uhlmann. Reduced Sigma point filters for the propagation of means and covariance through nonlinear transformations. Proceedings of the American Control Conference, (2002), 887-892. 